\title{
Power transmission risk assessment considering component condition
}

\author{
Lei GUO ( $ه)$, Qiwei QIU, Jian LIU, \\ Yu ZHOU, Linglei JIANG
}

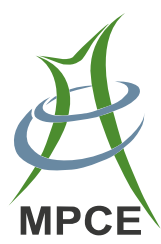

\begin{abstract}
This paper proposes a new method for power transmission risk assessment considering historical failure statistics of transmission systems and operation failure risks of system components. Component failure risks are integrated into the new method based on operational condition assessment of components using the support vector data description (SVDD) approach. The traditional outage probability model of transmission lines has been modified to build a new framework for power transmission system risk assessment. The proposed SVDD approach can provide a suitable mechanism to map component assessment grades to failure risks based on probabilistic behaviors of power system failures. Under the new method, both up-todate component failure risks and traditional system risk indices can be processed with the proposed outage model. As a result, component failure probabilities are not only related to historical statistic data but also operational data of components, and derived risk indices can reflect current
\end{abstract}

Received: 15 May 2013/Accepted: 21 September 2013/Published online: 21 Junuary 2014

(C) The Author(s) 2014. This article is published with open access at Springerlink.com

L. GUO, J. LIU, Y. ZHOU, North Subsection of State Grid

Corporation of China, Beijing 100053, China

$(\triangle)$ e-mail: stones_wall@163.com

J. LIU

e-mail: liu.jian@nc.sgcc.com.cn

Y. ZHOU

e-mail: zhou.yu@nc.sgcc.com.cn

Q. QIU, Shanghai Electric Power Company,

Shanghai 201400, China

e-mail: q2water@163.com

L. JIANG, Maintenance Company of Shanghai Electric Power

Company, Shanghai 200000, China

e-mail: ryandavidsharp@gmail.com operational conditions of components. In simulation studies, the SVDD approach is employed to evaluate component conditions and link such conditions to failure rates using up-to-date component operational data, including both on-line and off-line data of components. The IEEE 24-bus RTS-1979 system is used to demonstrate that component operational conditions can greatly affect the overall transmission system failure risks.

Keywords Risk assessment, Component failure risk, Outage probability, Condition assessment, Support vector data description

\section{Introduction}

With the continuous increase of energy demand, the accurate risk assessment of power systems is of great importance, since risks are increased when a power system is operated close to its stability limits due to distributed generation and market competition. With regard to power system assessment, higher risks lead to lower reliability, and vice versa. The probabilistic behaviors of power system failures are the root origin of risks [1], and an effective risk assessment model can provide quantitative risk indices to represent system reliability. Traditionally, only historical failure statistics are employed in power system risk assessment, however the overall system risk is also related to component operational conditions. When component failure risks change, the overall system risk varies accordingly. Incorporating component risks into the power system risk assessment can improve the accuracy and rationality of risk evaluations.

In the past decade, considerable efforts have been devoted to probabilistic risk assessment of power transmission systems and substation configurations. A widely 
used framework for power system risk assessment was reported in [1, 2], in which the approach, objective, application and economic cost were discussed in detail [3]. However, in this traditional framework, failure risks of components, such as transformers and circuit breakers, were not considered. Generally, the risk assessment of components in substations was performed separately [4-6]. As a result, there is a lack of a mechanism to convert component operational conditions into failure risks in the traditional framework. In [4], a risk assessment model of a combinative system in a transmission network and substations was proposed. Compared with the traditional framework, in which system risks of transmission networks and substation configurations are assessed separately, the method presented in [4] can evaluate system risks considering both transmission networks and substations by assessing new load curtailments at load points for each failure state. As an improvement, substations are no longer treated as a transmission node and substation configurations, and individual components, such as breakers and transformers, are linked to system risks by analyzing statistical data of substation components. However, component failure data are still based on historical statistics. Consequently, the impact of online component operational conditions cannot be integrated in risk evaluations.

A multi-objective risk assessment framework was presented in [7], and probabilistic indices for assessing realtime power system security levels were derived. However, operation risks of components were still not considered. A failure probability model was developed based on the evidential reasoning (ER) theory for overhead lines in [8], which can accurately reflect the impact of surroundings on failure probabilities. However, component outage rates were set as a fixed value, which was not linked to operational conditions of components. Based on the ER theory and the functional group decomposition principle, a contingency identification method for components was presented in [9].

However, in that research, component conditions, such as operational conditions and monitoring data, were not considered, and components were just treated as part of transmission lines. But actually, each component has its own failure risk, which is influenced by its operational condition. In practice, component condition assessment is usually conducted by experts or trained on-site engineers. As operational conditions could be affected by faults or environment, such as loading conditions and temperatures $[5,6]$, the failure probability of components is not fixed. Thus, the outage probability of transmission lines changes accordingly. As the component failure probability changes, the results of risk assessment are not fixed values as those of traditional risk assessment models [1], which should be determined by both operational conditions and historical data.
The support vector data description (SVDD) approach is developed for classification and evaluation with machine learning, which can be employed to aggregate diagnosis information $[10,11]$. In particular, regarding the probabilistic and uncertain behaviors of component failures, SVDD is a suitable solution for presenting evaluation of various failure conditions. Based on the outputs of SVDD component evaluation, system operators can obtain overall evaluations of studied components, which can be classified into different condition levels accordingly. The SVDD approach is capable of providing the most recent condition for components in power transmission systems. The objective of this paper is to develop a new risk assessment method for power transmission systems, in which component conditions are considered based on on-line and offline data. The method comprises of three parts: component evaluation, index transition and system risk evaluation. The proposed method employs SVDD for component risk assessment and the Monte Carlo (MC) simulation [1] for system state selections.

\section{SVDD approach to component condition assessment}

The SVDD approach is an one-class classification data description method which proposed by Tax [10]. By training with a set of certain samples, the distribution of target class can be obtained by SVDD, so the outliers can be divided. The SVDD approach can provide well distribution area and can be used in condition detection, fault diagnosis and multi-classification, etc. [12-14].

By applying SVDD approach to mechanical condition monitoring and fault diagnosis, machine conditions can be monitored only by using normal condition signals instead of abnormal condition signals. With the method, the machine set conditions (normal or abnormal) can be described by using quantitative indices, and the scientific decision-making basis for equipment management and predictive maintenance can be offered. The method is used to evaluate the condition of the key equipment in power transmission lines, and it correctly evaluates an abnormal condition of the equipment in time and contributes to a successful diagnosis of the incipient fault of a bolt crack.

As a data set containing $N$ data objects: $\left\{x_{i}, i=1,2, \ldots\right.$, $N$ \}, the basic concept of SVDD is trying to find a sphere with minimum volume, containing all (or most of) the data objects [10]. This is very sensitive to the most outlying object in the target data set. When one or a few very remote objects are in the training set, a very large sphere is obtained which will not represent the data very well. Therefore, [15] considered some data points outside the sphere and introduced slack variable $\xi_{i}\left(\xi_{i} \geq 0, i=1,2, \ldots, n\right)$. Of the 
sphere, described by center $a$ and radius $R$, the radius is minimized as follows:

$$
\begin{aligned}
& \min _{R} F(R, a)=R^{2}+C \sum_{i=1}^{n} \xi_{i} \\
& \text { s.t. }\left\{\begin{array}{r}
{\left[\varphi\left(x_{i}\right)-a\right]\left[\varphi\left(x_{i}\right)-a\right]^{\mathrm{T}} \leq R^{2}+\xi_{i}} \\
\xi_{i} \geq 0, \quad i=1,2, \ldots, n
\end{array}\right.
\end{aligned}
$$

where the variable $C$ gives the trade-off between the simplicity (or volume of the sphere) and the number of errors (number of target objects rejected); the function $\varphi$ is a nonlinear mapping function used for mapping objects into the high dimensional.

The dual form of (1) is written as:

$$
\begin{aligned}
& \max _{\alpha_{i}} L=\sum_{i=1}^{n} \alpha_{i} K\left(x_{i} \cdot x_{j}\right) \\
& \text { s.t. }\left\{\begin{array}{l}
\sum_{i=1}^{n} \alpha_{i}=1 \quad i=1,2, \ldots, n \\
0 \leq \alpha_{i} \leq C \quad i=1,2, \ldots, n
\end{array},\right.
\end{aligned}
$$

where $K\left(x_{i} \cdot x_{j}\right)$ is the kernel function which satisfies Mercer's theorem:

$K\left(x_{i} \cdot x_{j}\right)=\left[\varphi\left(x_{i}\right) \cdot \varphi\left(x_{j}\right)\right]$.

The kernel function implicitly maps the objects $x_{i}$ into some feature space and when a suitable feature space is chosen, a better and more tight description can be obtained. No explicit mapping is required, the problem is expressed completely in terms of $K\left(x_{i} \cdot x_{j}\right)$.

Finally, parameter $\alpha_{i}$ can be obtained, and $x_{i}$ satisfies $\alpha_{i}>0$, called the support vector. From the basic concept and definition of SVDD [11], the equation is obtained as follows:

$$
\begin{aligned}
R^{2}= & K\left(x_{k} \cdot x_{k}\right)-2 \sum_{i=1} \alpha_{i} K\left(x_{i} \cdot x_{k}\right) \\
& +\sum_{i, j} \alpha_{i} \alpha_{j} K\left(x_{i} \cdot x_{j}\right) \quad \forall x_{k} \in \mathrm{SV}<C,
\end{aligned}
$$

where $R^{2}$ is the distance to the center of the sphere (a); SV means support vector.

Based on (4), each support vector can provide the value of $R^{2}$. For the test sample $z$, assuming that:

$$
\begin{aligned}
D(a, z)= & K(z \cdot z)-2 \sum_{i=1} \alpha_{i} K\left(z \cdot x_{i}\right) \\
& +\sum_{i, j} \alpha_{i} \alpha_{j} K\left(x_{i} \cdot x_{j}\right) \quad i, j=1,2, \ldots, n .
\end{aligned}
$$

If $D(a, z) \leq R^{2}$, then $z$ is considered as the target, otherwise is considered as the outlier.

For illustration purposes, we define evaluation level as the SVDD result of each component. It corresponds to the relationships between component condition characteristic values and failure probability. For example, based on a transformer dissolved gas analysis (DGA) value, the SVDD evaluation level of this component is graded as 'good', 'normal', 'poor' or 'serious'. Therefore, each level equals an area of failure rate (from 0 to 1), afterwards, the component condition is considered in power transmission risk assessment.

\section{Power transmission system risk evaluation considering component risks}

In practice, power system risk assessment is concerned with two aspects: i.e., system adequacy and system security [1]. System adequacy mainly relates to the existence of sufficient facilities within a system to satisfy consumer load demands and system operational constraints, while system security relates to the ability of a system to respond to dynamic and transient disturbances arising within the system. Thus, security is associated with the response of a system to perturbations. As most of the risk assessments

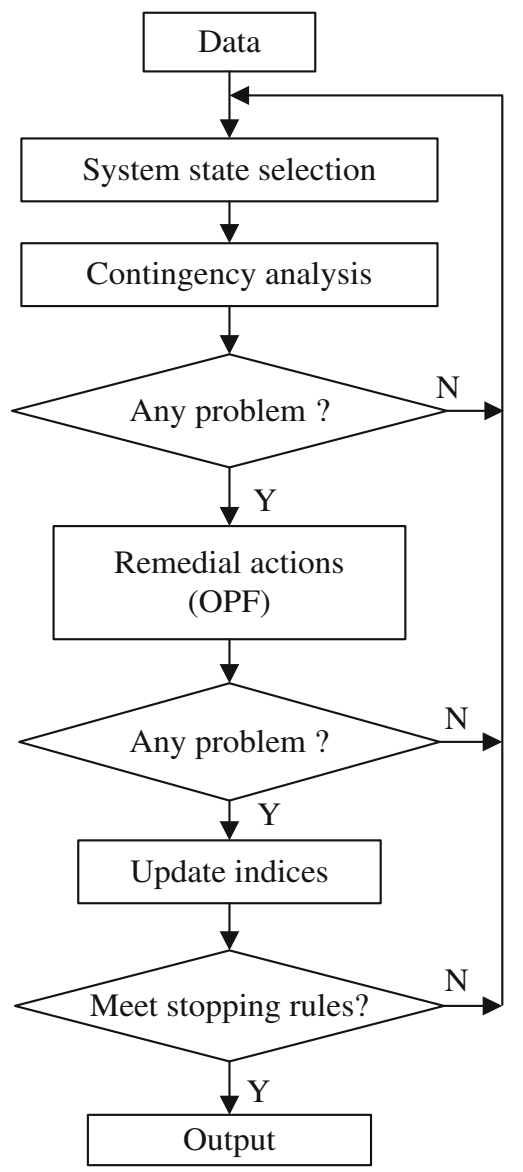

Fig. 1 Procedure of power transmission risk assessment 


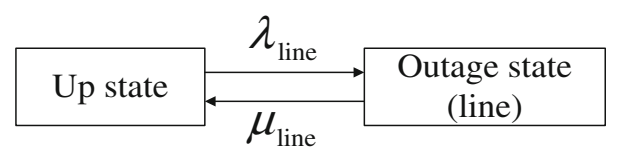

Fig. 2 State space diagram of a two-state repairable forced outage

carried out by utilities are in the domain of adequacy assessment [1], in this paper the system adequacy analysis is set as the risk assessment objective.

The basic procedure of power transmission system risk assessment is shown in Fig. 1 [1]. Firstly, a system sate is selected based on historical failure statistics. Then, the contingency analysis and optimal power flow (OPF) method are performed to identify whether a selected state causes any problems. Finally, risk indices are calculated. In this paper, procedures of outage modeling in system state selections have been modified using the updated outage model considering component failure risks.

\subsection{Traditional component failure models}

Traditionally, for power transmission system risk evaluation, only failures of transmission components are considered, whereas generating units are assumed to be $100 \%$ reliable. Key transmission components include overhead lines, cables, transformers, capacitors, and reactors. Generally, these components are represented by a two-state (up and down) model. Figure 2 shows the diagram of a basic two-state repairable forced outage, which can be used to describe a typical steady up-down-up cycle process.

The average unavailability of a transmission line in a long-term process is defined as follows [1]:

$P_{\text {line }}=\frac{\lambda_{\text {line }}}{\lambda_{\text {line }}+\mu_{\text {line }}}$,

$\left\{\begin{array}{l}\lambda_{\text {line }}=\frac{8,760}{M T T F_{\text {line }}}, \\ \mu_{\text {line }}=\frac{8,760}{M T T R_{\text {line }}}\end{array}\right.$

where $P_{\text {line }}$ is the outage probability of lines; $\lambda_{\text {line }}$ and $\mu_{\text {line }}$ are the line failure and repair rates (1/year), respectively; $M T T R_{\text {line }}$ and $M T T F_{\text {line }}$ are the mean time to repair (MTTR) (hours per year) and mean time to failure (MTTF) (hours/year) of lines, respectively.

The historical data are recorded over a one year period and subsequently the failure and repair rates can be derived based on MTTF and MTTR, respectively.

\subsection{Outage model integrating component failure risk}

A new outage model has been developed based on both historical failure statistics (IEEE RTS-79) [16] and

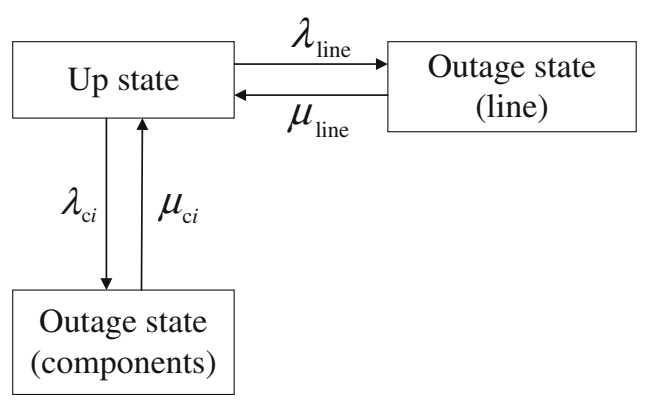

Fig. 3 State space diagram considering both line and component outages

component failure risks. In the traditional outage model, the forced outage probability of transmission lines is denoted by $P_{\text {line }}$. Based on (1), the planned outage model and Markov equations [1], the state space diagram considering both the line and component risks is shown in Fig. 3.

Applying the Markov method based on the state space diagram, the outage probabilities can be obtained as follows:

$$
\begin{aligned}
& P_{\mathrm{c} i}=\frac{\lambda_{\mathrm{c} i} \mu_{\mathrm{line}}}{\lambda_{\mathrm{c} i} \mu_{\text {line }}+\lambda_{\text {line }} \mu_{\mathrm{c} i}+\mu_{\text {line }} \mu_{\mathrm{c} i}}, \\
& P_{\text {up }}=\frac{\mu_{\text {line }} \mu_{\mathrm{c} i}}{\lambda_{\mathrm{c} i} \mu_{\text {line }}+\lambda_{\text {line }} \mu_{\mathrm{c} i}+\mu_{\text {line }} \mu_{\mathrm{c} i}}, \\
& P_{\text {line }}=\frac{\lambda_{\text {line }} \mu_{\mathrm{c} i}}{\lambda_{\mathrm{c} i} \mu_{\text {line }}+\lambda_{\text {line }} \mu_{\mathrm{c} i}+\mu_{\text {line }} \mu_{\mathrm{c} i}}, \\
& \left\{\begin{array}{l}
\lambda_{\mathrm{c} i}=\frac{8,760}{M T T F_{\mathrm{c} i}}, \\
\mu_{\mathrm{c} i}=\frac{8,760}{M T T R_{\mathrm{c} i}}
\end{array}\right.
\end{aligned}
$$

where $P_{\mathrm{up}}, P_{\text {line }}$ and $P_{\mathrm{c} i}$ are the probabilities of the up state, the historical line outage state and the failure risk-based outage state of the $i$ th component; $\lambda_{\text {line }}$ and $\lambda_{\mathrm{c} i}$ are the transition rates of historical line outages and component failure risk-based outage states; $\mu_{\text {line }}$ and $\mu_{\mathrm{c} i}$ are the recovery (repair) rates of the historical line outage and the component failure risk-based outage state (repairs/year); $M T T R_{\mathrm{c} i}$ and $M T T F_{\mathrm{c} i}$ are the MTTR and MTTF of component, respectively.

In Section 3, the condition assessment of components based on SVDD approach is used as an example to illustrate the procedures for calculating component failure risks. As discussed previously, the overall assessment of a component can be expressed using SVDD approach as a set of evaluation grades, and then component failure rates can be derived in association with the SVDD evaluation levels.

There are normally a number of components in the same transmission line or bus, and the line or bus fails when one 
of the main components fails. As a result, the maximum component failure risk value is selected to represent the overall failure probability of components in the same line. Considering failure risks of components, the transmission line outage probability integrating both historical failure statistics and component failure risks can be expressed in the following (13):

$P_{\mathrm{c}}=\max \left(P_{\mathrm{c} 1}, P_{\mathrm{c} 2}, \ldots, P_{\mathrm{c} n}\right)$,

$P_{\mathrm{L}}=P_{\text {line }}+P_{\mathrm{c}}=\frac{\lambda_{\text {line }} \mu_{\mathrm{c} i}+\lambda_{\mathrm{c} i} \mu_{\text {line }}}{\lambda_{\mathrm{c} i} \mu_{\text {line }}+\lambda_{\text {line }} \mu_{\mathrm{c} i}+\mu_{\text {line }} \mu_{\mathrm{c} i}}$,

where $P_{\mathrm{L}}$ is the overall outage probability of transmission lines; $P_{\mathrm{c}}$ is the maximum component failure risk among $n$ components in the same transmission line; $P_{\mathrm{c} n}$ is the outage probability of the $n$th component in the same line.

As shown in (13), it is assumed that the $i$ th component has the maximum failure risk.

\subsection{Load curve models and contingency analysis}

In this paper, for the state enumeration or state sampling method (nonsequential MC simulation), a nonchronological load duration curve is utilized. A single load curve is considered and loads at all buses are scaled proportionally to follow the shape of the given load curve. A multiple-step model is established to represent the load duration curve [1]. Regarding the contingency analysis on adequacy risk assessment, the capacity balance between the generation and the load demand is important. As a result, the DC power-flow-based contingency analysis is employed in this study, because it provides fast and sufficiently accurate real power flows following line outages for risk assessment, in which a large number of outage events are considered.

\subsection{Optimization models for load curtailment}

When an outage causes system problems, a special OPF model is used to reschedule generations and alleviate constraint violations. At the same time, load curtailment needs to be avoided if possible or the total load curtailment is required to be minimized if unavoidable. The objective function of an OPF model is to minimize the total load curtailment, whereas load curtailment at buses is the solution of the OPF model. The risk indices are then calculated based on load curtailments in selected system outage states and their probabilities of occurrence. To reduce the computational burden, the DC power-flow-based OPF model is usually employed in the adequacy risk assessment [1]. It can be expressed in the following equations:

$\min \sum_{i \in N D} C_{i}$,

$$
\text { s.t. }\left\{\begin{array}{l}
\boldsymbol{T}(S)=\boldsymbol{A}(S)(\boldsymbol{P G}-\boldsymbol{P D}+\boldsymbol{C}) \\
\sum_{i \in N G} P G_{i}+\sum_{i \in N D} C_{i}=\sum_{i \in N D} P D_{i} \\
P G_{i}^{\min } \leq P G_{i} \leq P G_{i}^{\max } \quad i \in N G \\
0 \leq C_{i} \leq P D_{i} \quad i \in N D \\
\left|T_{k}(S)\right| \leq T_{k}^{\max } \quad k \in L
\end{array}\right.
$$

where $i$ is the bus number; $C_{i}$ is the load curtailment at the $i$ th bus; $\boldsymbol{T}(S)$ is the real power flow vector in the outage state; $\boldsymbol{A}(S)$ is the relation matrix between real power flows and power injections in the outage state $S ; \boldsymbol{P G}$ and $\boldsymbol{P D}$ are the generation output and load power vectors, respectively; $\boldsymbol{C}$ is the load curtailment vector; $P G_{i}, P D_{i}, C_{i}$ and $T_{k}(S)$ are the elements of $\boldsymbol{P G}, \boldsymbol{P D}, \boldsymbol{C}$ and $\boldsymbol{T}(S)$, respectively; the subscript " $\min$ ", "max" are the limits, respectively; $N G, N D$ and $L$ are the sets of generation buses, load buses and branch circuits in a system.

The objective of the model is to minimize the total load curtailment while satisfying the power balance, DC power flow relationships and limits on line flows and generation outputs.

\subsection{Risk indices}

There are various risk indices, which are used for quantifying system risks. In practice, loss-of-load probability (LOLP) and expected demand not supplied (EDNS) are two most popular indices, which are employed in this research. LOLP indicates the probability of load loss caused by element capacity shortage (1/year). It can be expressed in the following equation:

$L_{\mathrm{LOLP}}=\sum_{x \in X} I_{\mathrm{f}}(x) P(x)$,

where $P(x)$ is the probability of system state $x ; I_{\mathrm{f}}(x)$ is a two valued function of system state $x$. If $x$

Table 1 Evaluation level corresponding to failure rate

\begin{tabular}{ll}
\hline Evaluation level & Failure rate (1/year) \\
\hline Good & $0-0.2$ \\
Normal & $0.2-0.8$ \\
Poor & $0.8-1.0$ \\
Serious & Outage \\
\hline
\end{tabular}

Table 2 Results of SVDD classification

\begin{tabular}{lrrrrr}
\hline Actual/evaluation & Good & Normal & Poor & Serious & Total \\
\hline Good & 39 & 7 & 4 & 0 & 50 \\
Normal & 5 & 42 & 2 & 1 & 50 \\
Poor & 0 & 1 & 26 & 3 & 30 \\
Serious & 0 & 2 & 1 & 27 & 30 \\
Total & 44 & 51 & 35 & & 130 \\
\hline
\end{tabular}




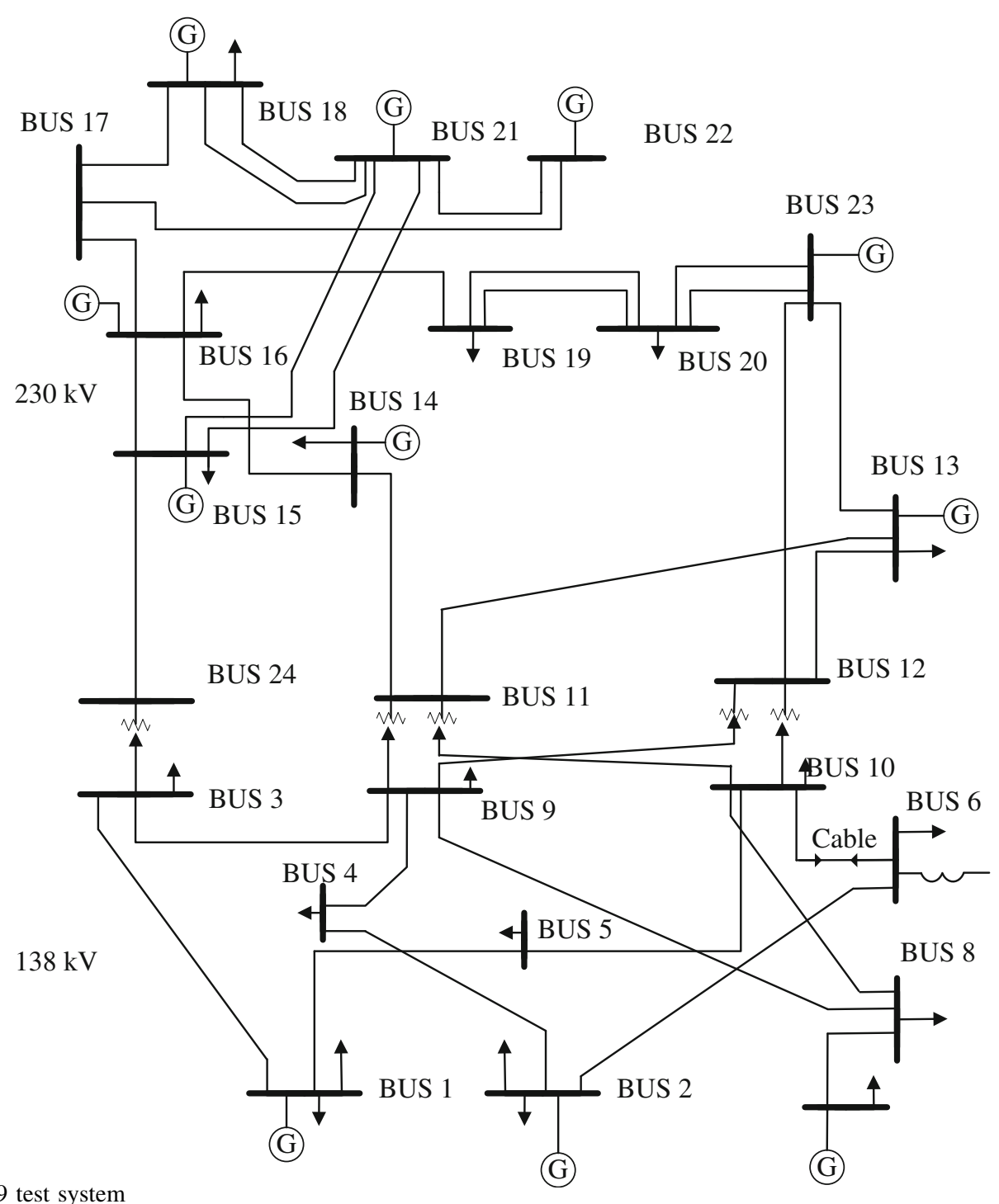

Fig. 4 IEEE RTS-79 test system

indicates the failure state, then $I_{\mathrm{f}}$ is equal to 1 , otherwise 0 .

EDNS denotes the average shortage of power supply per year (MW/year).

$L_{\mathrm{EDNS}}=\sum_{x \in X} I_{\mathrm{f}}(x) L_{\mathrm{C}}(x) P(x)$,

where $L_{\mathrm{C}}(x)$ represents the minimum load loss for recovery in the outage state $x$.

\section{Case study}

\subsection{Component failure risk mapping}

The condition assessment of transformers is used as an example to illustrate the procedures for calculating component failure risks. For other components, the procedures can be performed in a similar manner. As discussed previously, the overall assessment of a component can be expressed as a set of evaluation grades, then component failure rates can be derived in association with the SVDD evaluation levels. For illustration purposes, Table 1 lists the corresponding relationships between evaluation levels and failure rates. For example, based on historical statistics or operation experience, the failure rate of a component is 0.14 per year, and then the SVDD evaluation level of this component is graded as 'good'.

Table 1 only gives reference values for illustration purposes, and in practice, this table may be modified based on operation situations and historical statistics analysis. It is defined that the SVDD evaluation grade, that is 'serious', 'poor', 'normal' or 'good', with the maximum value is treated as the final evaluation grade of a component. Using this mapping table, the failure rate $\lambda_{\mathrm{c}}$ can be derived by the proposed SVDD approach. Likewise, the repair rates $\mu_{\mathrm{c}}$ can 
Table 3 Case I: LOLP and ENDS results at different sampling frequencies

\begin{tabular}{lllll}
\hline $\begin{array}{l}\text { Sampling } \\
\text { times }\end{array}$ & $\begin{array}{l}\text { LOLP } \\
(\%)\end{array}$ & $\begin{array}{l}\text { LOLP } \\
\text { considering } \\
\text { component }\end{array}$ & $\begin{array}{l}\text { EDNS } \\
\text { (MW/ } \\
\text { year) }\end{array}$ & $\begin{array}{l}\text { EDNS } \\
\text { considering } \\
\text { component }\end{array}$ \\
\hline 10,000 & 8.72 & 9.38 & 14.29 & 14.42 \\
20,000 & 8.69 & 9.25 & 14.62 & 14.39 \\
\hline
\end{tabular}

be defined similarly. In the meantime, $\lambda_{\text {line }}$ and $\mu_{\text {line }}$ can be derived from historical statistic data, and finally the outage probability of components can be calculated by using (8).

\subsection{SVDD-based component failure risk evaluation}

In this paper, 260 sets of transformer monitoring data sample are adopted. These samples are acquired from the DGA monitoring of transformers. Thereinto, 130 sets are used for training (50 sets are in good condition, 50 sets are normal, 30 set are poor and 30 sets are serious) and others are used for testing. By adopting the SVDD approach, the evaluation results are listed in Table 2.

In the evaluation results, the total accuracy is about $84 \%$. The accuracy of 'good', 'normal', 'poor' and 'serious' are $78 \%, 84 \%, 87 \%$ and $90 \%$, respectively.

Based on the mapping relations listed in Table 1, the failure rate of a component can be generated randomly between the areas. However, the mapping relation is only for illustrating reference values, those values can be defined based on the practice or experience.

\subsection{System risk assessment: Case I}

In this case study, the IEEE RTS-79 system is employed as the test system [15] as shown in Fig. 4. In the IEEE RTS79 test system, the load model gives hourly loads for one year on a per unit basis, expressed in chronological fashion so that daily, weekly and seasonal patterns can be modeled. The generating system contains 32 units, ranging from 12 to $400 \mathrm{MW}$. The transmission system contains $24 \mathrm{load} / \mathrm{gener}-$ ation buses connected by 38 lines or autotransformers at two voltage levels, i.e. $138 \mathrm{kV}$ and $230 \mathrm{kV}$. The transmission system includes cables, lines on a common right of way, and lines on a common tower. The transmission system data include the line length, impedance, ratings, and reliability data.

In MC simulations different sampling frequencies lead to different convergences, therefore the sampling frequencies of the MC simulation are set with different values. As a result, the derived risk index values at different sampling frequencies are presented in Table 3 .

It is assumed that all the transformers in the system are operated under a 'normal' condition, and the component
Table 4 Case II: LOLP and ENDS results at different sampling frequencies

\begin{tabular}{lll}
\hline $\begin{array}{l}\text { Sampling } \\
\text { times }\end{array}$ & $\begin{array}{l}\text { LOLP considering } \\
\text { component }\end{array}$ & $\begin{array}{l}\text { EDNS considering } \\
\text { component }\end{array}$ \\
\hline 10,000 & 12.01 & 16.68 \\
20,000 & 10.65 & 15.28 \\
\hline
\end{tabular}

failure rates are set as a random value between 0.2 and 0.8 randomly according to Table 1 .

Compared with the results without considering component risks the LOLP and EDNS have rarely increased.

\subsection{System risk assessment: Case II}

In Case II, the component failure rates are set higher than that of Case I and all system components are assumed to be operated under a 'poor' condition. The component failure risks are generated between 0.8 and 1.0 mapping to the 'poor' level for each line. Different sampling frequencies are also compared, as shown in Table 4.

In Case II, it is clear that when evaluation levels of all transformers are changed from 'normal' to a worse level like 'poor', LOLP increases significantly. Compared with these of Case I, LOLP considering component risks increases nearly by $15 \%$, while ENDS considering components is raised by $6 \%$.

\subsection{System risk assessment: Case III}

In this case, only the component between BUS 3 and BUS 24 is set as outage, while other components in the system are considered as 'normal'. According to Table 1, the probability of the outage component is $100 \%$, and failure rates of other components are between 0 and 0.2. Different sampling frequencies are applied and its results concerning LOLP and ENDS are listed in Table 5. Compared with Case II, LOLP and EDNS are 8 and 3\% more than those of Case I, which indicates that when a transformer is working under a 'serious' condition, the overall system risk increases. However, the risks are less than those in Case II, which indicates that when failure risks of several components change from 'normal' to a 'poor' or 'serious' grade, the risk values

Table 5 Case III: LOLP and ENDS results at different sampling frequencies

\begin{tabular}{lll}
\hline $\begin{array}{l}\text { Sampling } \\
\text { times }\end{array}$ & $\begin{array}{l}\text { LOLP considering } \\
\text { component }\end{array}$ & $\begin{array}{l}\text { EDNS considering } \\
\text { component }\end{array}$ \\
\hline 10,000 & 9.81 & 14.70 \\
20,000 & 9.92 & 14.82 \\
\hline
\end{tabular}




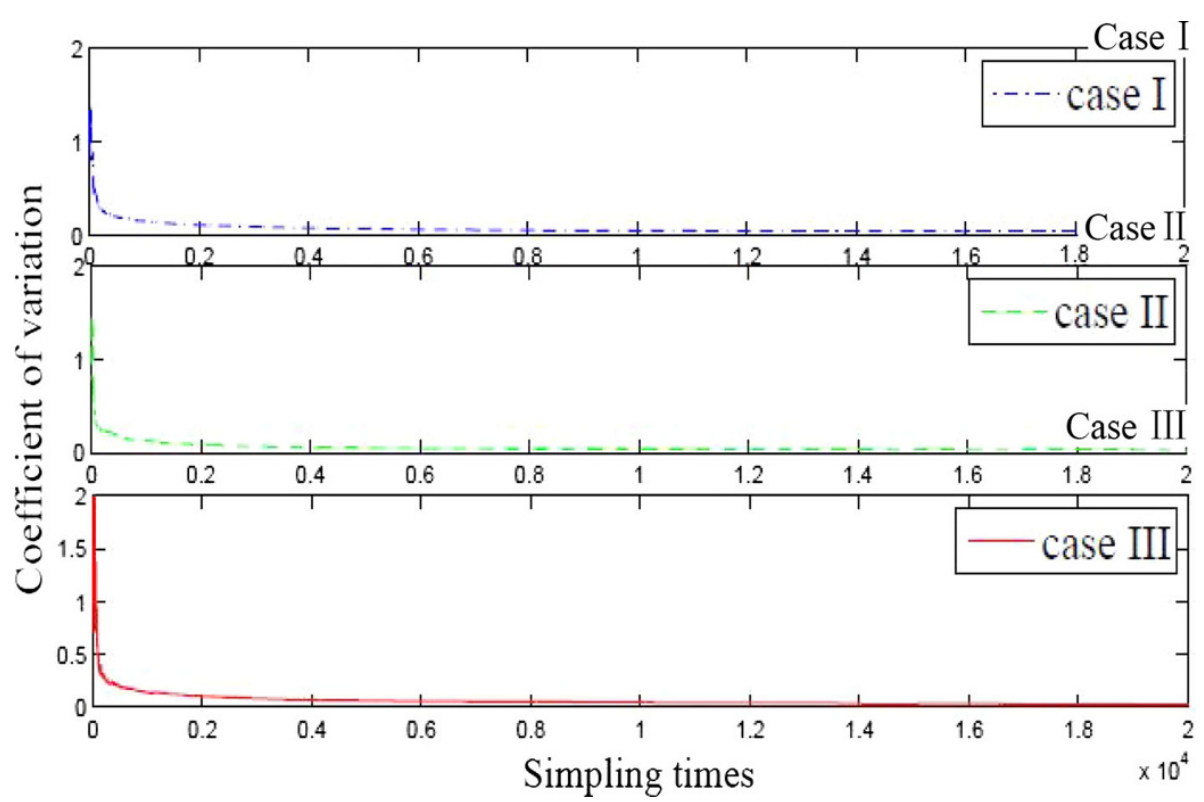

Fig. 5 Convergence variation of EDNS

increase much more than the situation that only one component is in the 'serious' grade.

The variation convergence curves of EDNS in three cases are illustrated in Fig. 5, which shows that when the sampling frequency is over 20,000 times, the variation convergence is relatively small under 0.2 . It means that, for three cases the derived coefficients are reliable when the sampling frequency is over 20,000 times.

\section{Conclusion}

A new method for transmission system risk assessment considering component monitoring data is proposed. The proposed SVDD-based approach can provide a suitable mechanism to map component evaluation grades to failure risks based on the probabilistic behaviors of power system failures. Using the new method, both up-to-date component condition status and traditional system risk indices can be processed with the developed outage model. In this study, transformer DGA data have been used to calculate component failure risks. The simulation results indicate that transmission system risks are affected not only by component operational conditions, but also by historical statistics data. In case studies, the implementation procedures of component risk evaluation using SVDD and system risk assessment are demonstrated.

Open Access This article is distributed under the terms of the Creative Commons Attribution License which permits any use, distribution, and reproduction in any medium, provided the original author(s) and the source are credited.

\section{References}

[1] Li WY (2005) Risk assessment of power systems: models, methods, and applications. Wiley, Hoboken

[2] Billinton R, Li W (1994) Reliability assessment of electric power systems using Monte Carlo methods. Plenum, New York

[3] Ghajar RF, Billinton R (2006) Economic costs of power interruptions: a consistent model and methodology. Int $\mathrm{J}$ Electr Power Energy Syst 28(1):29-35

[4] Li WY, Lu JP (2005) Risk evaluation of combinative transmission network and substation configurations and its application in substation planning. IEEE Trans Power Syst 20(2): $1144-1150$

[5] Tang WH, Spurgeon K, Wu QH et al (2004) An evidential reasoning approach to transformer condition assessments. IEEE Trans Power Deliv 19(4):1696-1703

[6] Shintemirov A, Tang WH, Wu QH (2010) Transformer winding condition assessment using frequency response analysis and evidential reasoning. IET Electr Power Appl 4(3):198-212

[7] Xiao F, McCalley JD (2009) Power system risk assessment and control in a multiobjective framework. IEEE Trans Power Syst 24(1):78-85

[8] Zhang GH, Duan MY, Zhang JH et al (2009) Power system risk assessment based on the evidence theory and utility theory. Automat Electr Power Syst 33(23):1-12 (in Chinese)

[9] Song Y, Wang CS (2008) N-K contingency identification method under double failure incident based on evidence theory and functional group decomposition. Proc Chin Soc Electr Eng 28(28):47-53 (in Chinese)

[10] Tax DMJ (2004) Support vector data description. Mach Learn $54(1): 45-66$

[11] Tax DMJ, Duin RPW (2000) Data description in subspaces. In: Proceedings of the 15th international conference on pattern recognition (ICPR'00), vol 2, Barcelona, Spain, 3-7 Sept 2000, pp 672-675

[12] Zhui XK, Yang DG (2007) Multi class support vector domain description for pattern recognition based on a measure of expansibility. Acta Electron Sin 37(3):464-469 (in Chinese) 
[13] Tang MZ, Wang YB, Yang HC (2011) Modified support vector data description for fault diagnosis. Control Decis 26(7):967-972 (in Chinese)

[14] Li LJ, Han J, Hao W et al (2005) Condition evaluation for mechanical equipment by means of support vector data description. J Mech Sci Technol 24(12):1426-1429 (in Chinese)

[15] Tax DMJ, Duin RPW (1999) Support vector domain description. Pattern Recogn Lett 20(11-13):1191-1199

[16] IEEE RTS Task Force of APM Subcommittee (1979) IEEE reliability test system. IEEE Trans Power App Syst 98(6):2047-2054

Lei GUO was born in 1984. He obtained his Ph.D. in Electrical Engineering from Zhejiang University, Hangzhou, China, in 2012. He is currently working in the North Subsection of State Grid Corporation of China, Beijing, China. His research interests include power system stability, power grid planning, intelligent information processing technology and its application in power systems.
Qiwei QIU was born in 1978. He is currently working in the Shanghai Electric Power Company. His research interests include power transmission technology and management.

Jian LIU was born in 1979. He obtained his master degree in Tsinghua University, Beijing, China, in 2006. He is currently working in the North Subsection of State Grid Corporation of China, Beijing, China. His research interests include power system stability and power grid dispatching.

Yu ZHOU was born in 1983. He is currently working in the North Subsection of State Grid Corporation of China, Beijing, China. His research interests include power system stability and power grid dispatching.

Linglei JIANG was born in 1980. He is currently working in the Maintenance Company of Shanghai Electric Power Company. His research interests include power transmission technology and management. 